\title{
Antagonism against Vibrio cholerae by diffusible substances produced by bacterial components of the human faecal microbiota
}

\author{
S.H. SILVA, E.C. VIEIRA*, R.S. DIAS† and J.R. NICOLI \\ Departmento de Microbiologia and *Departamento de Bioquímica-Imunologia, Instituto de Ciências Biológicas, \\ Universidade Federal de Minas Gerais and †Fundação Ezequiel Dias, Belo Horizonte MG, Brazil
}

\begin{abstract}
Cholera vibrios sometimes survive, probably in low-level silent populations, in the small intestine of chronic carriers or pass through the gastrointestinal tract of a few individuals without causing diarrhoea or colonisation. To understand these situations, the present study used plate cultures (ex-vivo test) to investigate the frequency of appearance of an inhibitory halo against Vibrio cholerae produced by faecal specimens from 92 healthy volunteers (40 females, 52 males) aged 4-61 years. The frequency of inhibitory halo was $\mathbf{2 0 . 6 \%}$ in the whole group. An apparently higher percentage $(\mathbf{2 7 . 3 \% )}$ was observed in the age range 20-40 years when compared with the range 4-19 years $(10.7 \%)$, but not the range $41-61$ years $(20.0 \%)$. Frequency was significantly higher in males $(30.8 \%)$ than females $(7.5 \%)$. The dominant microbiota of a volunteer whose faeces produced an inhibitory halo was isolated by plate culture of decimal dilutions in an anaerobic chamber. Potential isolates of 26 apparently different morphologies were associated with germ-free NIH mice. One week later, the inhibitory test showed an antagonistic halo around the faeces from the associated animals, but not from the axenic mice. Of the 26 bacteria isolated, two (Lactobacillus sp. and Peptostreptococcus sp.) produced a compound antagonistic against $\boldsymbol{V}$. cholerae in an in-vitro assay. When biassociated with germ-free mice those strains eliminated the vibrio from the intestinal ecosystem in c. 5 days.
\end{abstract}

\section{Introduction}

The potent resistance of the gastrointestinal ecosystem of conventional rodents to colonisation with human enteric pathogens such as Shigella flexneri or Vibrio cholerae has been known for many years [1]. In contrast, the resident digestive microbiota of the major proportion of the human population is not so efficient. However, cholera vibrios sometimes survive, probably in the low-level repressed populations, in the small intestine of chronic human carriers [2] or pass through the gastrointestinal tract of a few individuals without causing diarrhoea or colonisation [3]. In the latter two situations, various possible immunological, physiological or microbiological factors may explain this resistance. To evaluate the role of microbial interference, the present study investigated the frequency of

Received 22 Feb. 2000; revised version received 2 June 2000; accepted 29 June 2000.

Corresponding author: Dr J.R. Nicoli (e-mail: jnicoli@, mono.icb.ufmg.br). appearance of an inhibitory halo against $V$. cholerae produced by the faeces of healthy volunteers (ex-vivo assay) and the bacterial components from the dominant faecal microbiota possibly responsible for this phenomenon were isolated.

\section{Patients and methods}

\section{Patients}

The faecal specimens were obtained from 92 healthy volunteers (40 females, 52 males; aged 4-61 years) who had not taken antibacterial agents or other drugs for at least 1 month before the assays.

\section{Mice}

Germ-free NIH mice (Taconic, Germantown, USA) were used as microbial receptors. The animals were housed in flexible plastic isolators (Class Biologically Clean, Madison, WI, USA) and were fed a commercial autoclavable diet (Nuvital, Curitiba, Brazil). The experiments with germ-free mice associated with 
bacterial components from human faecal microbiota were performed in micro-isolators (UNO Roestvastaal BV, Zevenar, The Netherlands).

\section{Detection of inhibitory diffusible substance by in- vitro and ex-vivo tests}

The in-vitro assay for an inhibitory diffusible compound was performed by the double-layer method. Bacteria that could potentially produce the inhibitory substance were spot inoculated on the surface of Brain Heart Infusion (BHI) Agar or deMan, Rogosa and Sharp (MRS) Agar (Difco, Detroit, USA) on a petri dish. After incubation at $37^{\circ} \mathrm{C}$ for $48 \mathrm{~h}$, the cells were killed by exposure to chloroform for $30 \mathrm{~min}$. Residual chloroform was allowed to evaporate and the petri dish was overlaid with $3.5 \mathrm{ml}$ of BHI soft agar (0.7\%) which had been inoculated with $0.2 \mathrm{ml}$ of an 18-h culture of $V$. cholerae. After incubation for $24 \mathrm{~h}$ at $37^{\circ} \mathrm{C}$, the dish was evaluated for the presence of an inhibitory halo.

For the ex-vivo assay, recently collected faeces from the human volunteers (sample standardised at $0.5 \mathrm{~g}$ ) or from mice $(10 \mathrm{mg})$ were placed on petri dishes containing thiosulphate-citrate-bile-sucrose (TCBS) agar and incubated for $48 \mathrm{~h}$ at $4^{\circ} \mathrm{C}$. After incubation, the faeces were treated with chloroform as described above. Then, the plates were spread with $0.1 \mathrm{ml}$ of a 10-fold dilution from an 18-h $V$. cholerae culture and incubated at $37^{\circ} \mathrm{C}$ for $24 \mathrm{~h}$. The plates were then checked for the presence of an inhibitory halo around the faecal inoculum. The $V$. cholerae non-enterotoxigenic, non-O1 strain 1609 used in the assay is a clinical isolate (male, 51 years old) obtained from the FIOCRUZ (Oswaldo Cruz Fundation), Rio de Janeiro, Brazil.

\section{Screening of human faecal bacteria producing an inhibitory substance against $V$. cholerae}

The dominant faecal microbiota from a patient (male, 37 years old) whose faecal sample produced an inhibitory halo against $V$. cholerae was obtained by decimal dilution in an anaerobic chamber containing an atmosphere of $\mathrm{N}_{2}, 85 \%, \mathrm{H}_{2}, 10 \%, \mathrm{CO}_{2} 5 \%$ (Forma Scientific, Marietta, USA). Samples diluted from 1 in $10^{7}$ to 1 in $10^{9}$ were spread on to blood agar supplemented with haemin $10 \mu \mathrm{g} / \mathrm{ml}$ and menadione $1 \mu \mathrm{g} / \mathrm{ml}$. After incubation at $37^{\circ} \mathrm{C}$ for 7 days, the different colony morphologies were isolated and submitted to microscopic examination and respiratory and biochemical tests. Biochemical and physiological tests were also performed with the API 20A, API 20STREP and API 50CH identification system kits (bioMérieux, Marcy-l'Etoile, France) for anaerobes, streptococci and carbohydrate metabolism, respectively. The BBL Crystal ${ }^{\mathrm{TM}}$ E/NF identification system kit (Becton Dickinson Microbiology Systems, Cockeysville, USA) was used for enterobacteria.

\section{Association of germ-free mice with human faecal bacteria}

The isolates were grown separately on BHI agar for $48 \mathrm{~h}$ at $37^{\circ} \mathrm{C}$ in the anaerobic chamber, washed off with sterile buffered saline, and immediately associated with germ-free mice. A $0.5-\mathrm{ml}$ sample of these suspensions was used to inoculate each animal by the orogastric route. Ex-vivo inhibitory assays were performed 7 days after the association of the germ-free mice with the different combinations of human faecal bacteria. Challenge with $V$. cholerae was performed intragastrically with a $0.1 \mathrm{ml}$ suspension containing c. $10^{8}$ viable bacterial cells when an inhibitory halo was observed around the faeces of the gnotoxenic mice. Germ-free mice challenged with the same pathogenic bacteria were used as the control group.

\section{V. cholerae counts}

After oral challenge, fresh faeces from gnotoxenic mice were collected daily and decimal dilutions were made for viable counts on TCBS agar spread plates after incubation for $24 \mathrm{~h}$ at $37^{\circ} \mathrm{C}$.

\section{Statistical analysis}

Inhibitory halo frequencies were compared by Fisher's exact test. Results were considered to be significantly different only when $\mathrm{p}<0.05$. Statistical analysis was performed with the EPISTAT software package (T.L. Gustafson, Round Rock, USA).

\section{Results and discussion}

Colonisation resistance is the ability of the normal microbiota associated with man and animals to protect these hosts against the unwanted establishment of pathogens [4]. The production of antimicrobial compounds by components of the normal microbiota is probably one of the most important mechanisms responsible for this antagonistic phenomenon. Although the presence of these antimicrobial metabolites has been demonstrated in vitro, it is unclear whether they are produced or have activity in vivo. It is known that the antagonism which can be seen in vitro between bacteriocinogenic and sensitive strains is not expressed in vivo in the digestive tract of gnotobiotic mice. Moreover, for some data obtained in the gastrointestinal ecosystem of gnotobiotic animals, a sensitive strain exerted a barrier effect against a strain which produced a bacteriocin [5]. On the other hand, Ramare et al. [6] demonstrated the presence of an antibacterial substance in the faeces of gnotobiotic rats mono-associated with a human Peptostreptococcus sp. This compound appeared to be produced through the concerted action of the host trypsin and a substance produced in vivo by the Peptrostreptococcus sp. This last example shows the active participation of the host in a bacterial 
interaction occurring in the digestive ecocystem and demonstrates the importance on an in vivo model, such as gnotobiotic animals, to study these inter-relationships.

Fig. 1 shows the frequency of an inhibitory halo against $V$. cholerae produced by the faeces of human volunteers. The halo diameter ranged from 1.5 to $4.0 \mathrm{~cm}$ (mean size $2.53 \mathrm{SD} 0.72 \mathrm{~cm}$ ) and the criterion for the determination of the results was the presence (positive) or absence (negative) of the inhibitory zone, independently of its size. An example of this antagonism is shown in Fig. 2. All the human subjects in the experiments $(\mathrm{n}=92)$ were healthy volunteers submitted to routine examination of their faeces. Some of them were asymptomatic carriers of Ascaris lumbricoides $(\mathrm{n}=19)$, Entamoeba histolytica (12), Ent. coli (7), Enterobius vermicularis $(\mathrm{n}=2)$ or Hymenolepis nana (1). However, there was no correlation between the presence of these parasitic protozoans and worms and the formation of an inhibitory halo against $V$. cholerae. The frequency of inhibitory halo was $20.6 \%$ in the whole group and, as expected, was much lower than the frequency $(85 \%)$ found with rodent faeces [7]. An apparently $(p=0.079)$ higher percentage $(27.3 \%)$ was observed in the age range $20-$ 40 years when compared with the age range 4-19 years $(10.7 \%)$, but not $(\mathrm{p}=0.385)$ when compared with the range 41-61 years $(20.0 \%)$. Frequency was significantly higher $(\mathrm{p}=0.005)$ in males $(30.8 \%)$ that females $(7.5 \%)$. At present these results cannot be explained. As regards the influence of age, a microbial digestive ecosystem in an installation stage could be a hypothesis for the lower frequency of antagonism found for the younger subjects. However, this explanation can be used only for the very young age group (0-

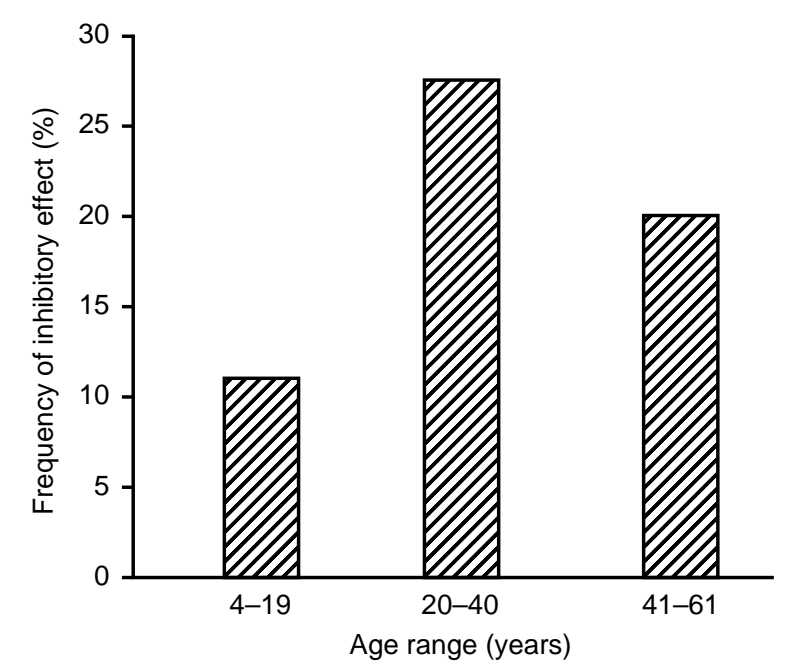

Fig. 1. Frequency of an inhibitory halo against $V$. cholerae caused by a diffusible substance from the faeces of human volunteers in different age ranges: 419 years $(\mathrm{n}=28) ; 20-40$ years $(\mathrm{n}=44) ; 41-61$ years $(\mathrm{n}=20)$.

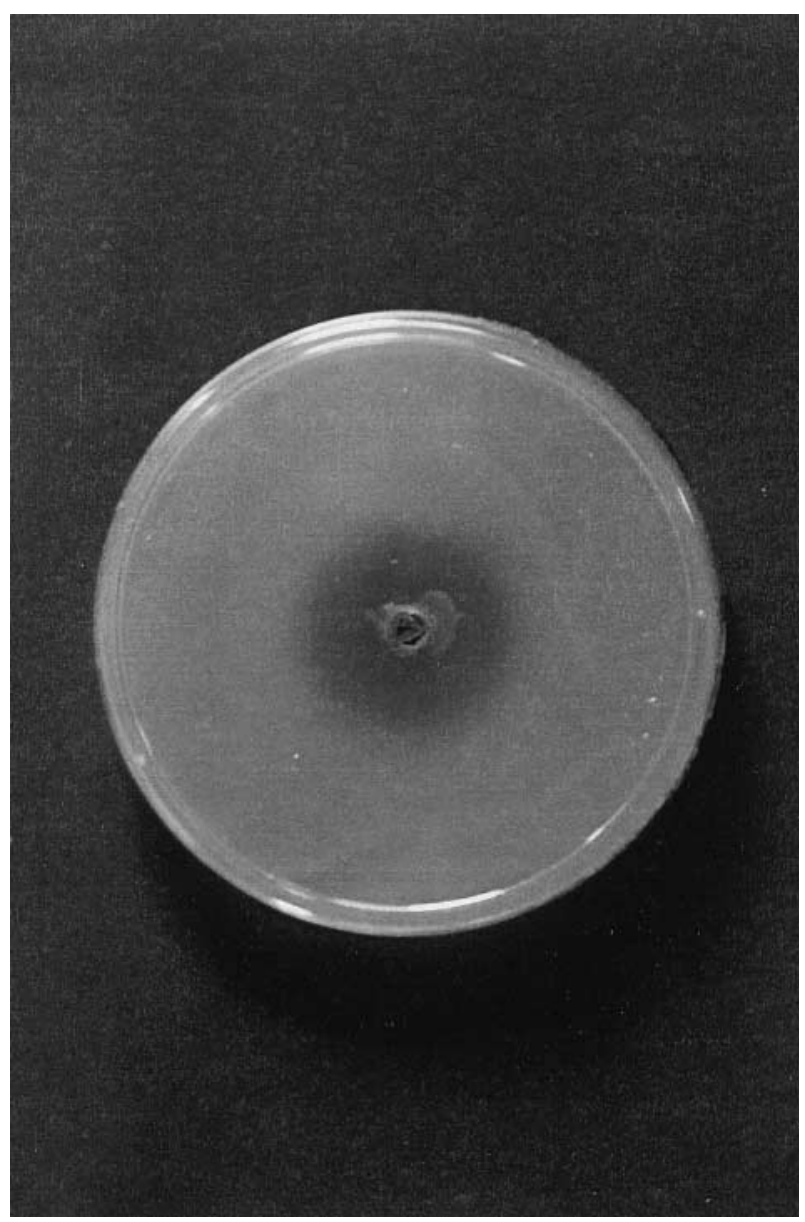

Fig. 2. Inhibitory test against $V$. cholerae on TCBS cholera medium with faeces from a human volunteer (female, 26 years old).

2 years). On the basis of the above data, a 37-year-old male volunteer whose faeces produced an inhibitory halo was selected as faecal donor for the isolation of bacteria responsible for the antagonism against $V$. cholerae. A stable and repeatedly observed inhibitory halo was the criterion in the choice of this subject. The dominant faecal microbiota was isolated by decimal dilution and culture in the anaerobic chamber. Bacteria of 26 apparently different morphological types (14 strict anaerobes and 12 facultative species) were associated with germ-free NIH mice. One week later, the ex-vivo assay showed an antagonistic halo around the faeces of the associated animals, but not the axenic animals. After oral challenge of the gnotoxenic mice with $V$. cholerae, the bacterial pathogen was eliminated from the faeces in 7 days. In an in-vitro inhibitory assay, two of the 26 bacteria showed antagonism against $V$. cholerae. Two groups of germ-free mice were then mono-associated with each of the inhibitory bacteria, one group was associated with the 24 remaining bacteria and one germ-free group was used as control. Fig. 3 shows that after oral challenge, $V$. cholerae was eliminated in 5 days in both bi-associated (one human bacterium and V. cholerae) groups and also 


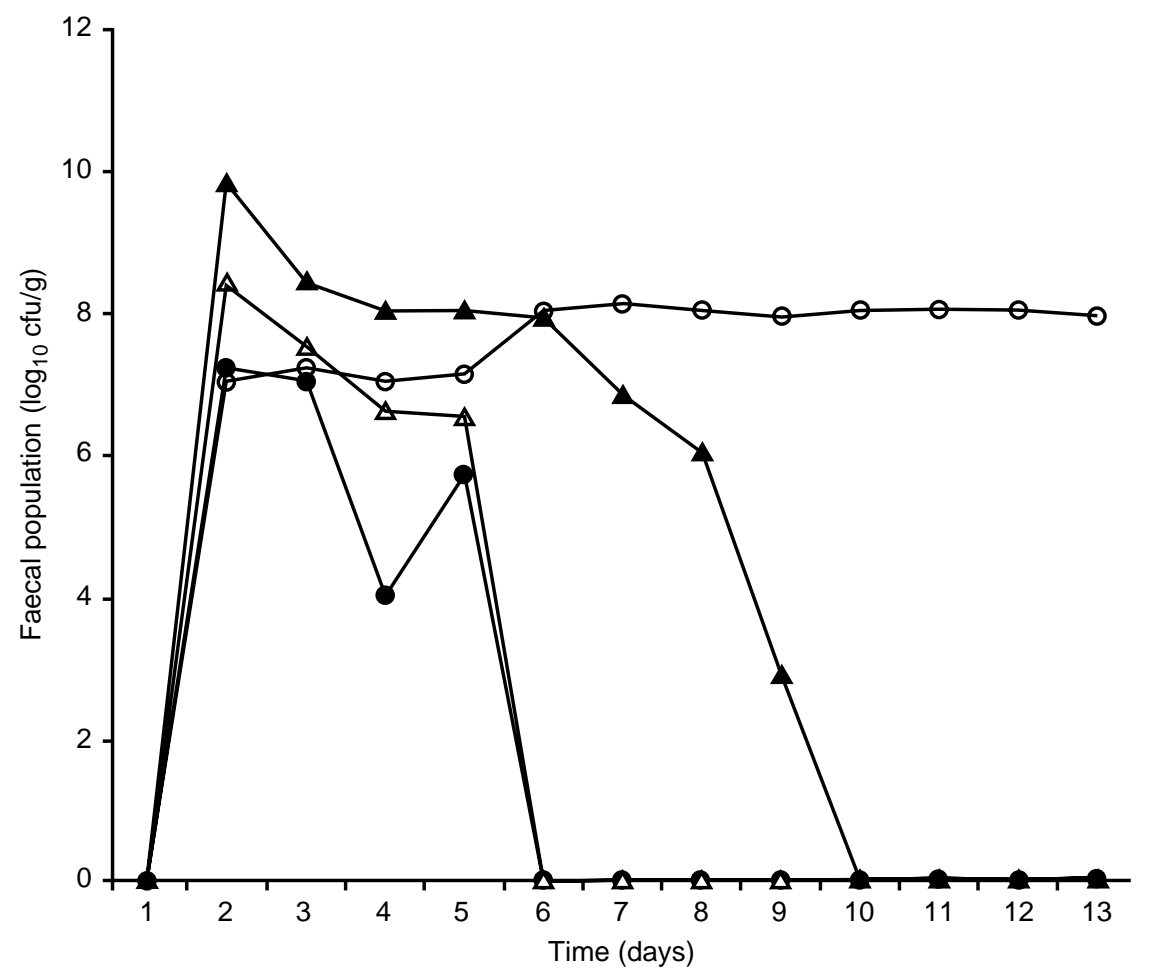

Fig. 3. Faecal population levels of $V$. cholerae in orally challenged germ-free mice $(\bigcirc)$ or in gnotoxenic mice previously associated with a Lactobacillus sp. (๑), a Peptostreptococcus sp. $(\triangle)$ or 24 human faecal bacteria $(\boldsymbol{\Delta})$.

in mice previously associated with the 24 bacteria but after a longer time period (10 days). In the last group, the 24 bacteria which did not show an inhibitory halo probably used another mechanism to eliminate the bacterial pathogen, such as competition for nutrients or adhesion site. These mechanisms are generally less efficient than antagonistic substances in the elimination of a target strain. Colonisation of the digestive ecosystem by the vibrio was obtained only in germfree mice. The two human bacteria were partially identified as a Lactobacillus sp. and a Peptostreptococcus sp., respectively.

These preliminary results suggest that some members of the human population have a digestive ecosystem resistant to the multiplication of $V$. cholerae through the production of inhibitory diffusible compounds. The data also show that the rapid elimination of the vibrio was exerted by more than one bacterial component at any given moment.

This work was supported by grants from Fundação de Amparo à Pesquisa do Estado de Minas Gerais (FAPEMIG) and Conselho
Nacional de Desenvolvimento Científico e Tecnológico (CNPq). We thank Maria Gorete Barbosa Ribas for valuable technical help.

\section{References}

1. Freter R. Experimental enteric shigella and vibrio infections in mice and guinea pigs. J Exp Med 1956; 104: 411-418.

2. Gorbach SL, Banwell JG, Pierce NF, Chalterjee BD, Mitra RC. Intestinal microflora in a chronic carrier of Vibrio cholerae. $J$ Infect Dis 1970; 121: 383-390.

3. Mosley WH, Beneson AS, Barui R. A serological survey of cholera antibodies in rural East Pakistan 1. The distribution of antibody in the control population of a cholera-vaccine field-trial area and the relation of antibody titre to the pattern of endemic cholera. Bull World Health Organ 1968; 38: 327-334.

4. Berg RD. The indigenous gastrointestinal microflora. Trends Microbiol 1996; 4: 430-435.

5. Duval-Iflah Y, Raibaud P, Rousseau M. Antagonisms among isogenic strains of Escherichia coli in the digestive tracts of gnotobiotic mice. Infect Immun 1981; 34: 957-969.

6. Ramare F, Nicoli J, Dabard J et al. Trypsin-dependent production of an antibacterial substance by a human Peptostreptococcus strain in gnotobiotic rats and in vitro. Appl Environ Microbiol 1993; 59: 2876-2883.

7. da Silva SH, Vieira EC, Nicoli JR. Antagonism against Vibrio cholerae by bacterial diffusible compound in the fecal microbiota of rodents. Rev Microbiol 1998; 29: 228-233. 